\title{
Binarity as the Solution to the Stellar Evolution Enigma Posed by NGC 6791
}

\author{
Giovanni Carraro $^{1}$ and Omar G. Benvenuto ${ }^{2,3}$ \\ ${ }^{1}$ Dipartimento di Fisica e Astronomia Galileo Galilei, Universitá di Padova, Vicolo Osservatorio 3, I-35122, Padova, Italy; giovanni.carraro@unipd.it \\ ${ }^{2}$ Instituto de Astrofísica de La Plata, CCT-CONICET-UNLP and Facultad de Ciencias Astronómicas y Geofísicas, \\ Universidad Nacional de La Plata Paseo del Bosque S/N, B1900FWA La Plata, Argentina; obenvenu@fcaglp.unlp.edu.ar \\ Received 2017 February 27; revised 2017 May 3; accepted 2017 May 3; published 2017 May 18
}

\begin{abstract}
Binary evolution is investigated as the source for the extreme horizontal branch (EHB) stars in the old and metalrich open cluster NGC 6791. Employing an updated version of our binary stellar evolution code, we demonstrate that EHB stars naturally emerge from the common-envelope phase. In sum, the binary model reproduces the observed $\left(T_{\text {eff }}, \log g\right.$ ) and temporal properties of the EHB overdensity tied to NGC 6971, without needing an ad hoc and anomalous mass-loss prescription.
\end{abstract}

Key words: binaries: general - open clusters and associations: individual (NGC 6791) - subdwarfs

\section{Introduction}

NGC 6791 is a metal-rich and old Galactic open cluster $([\mathrm{Fe} / \mathrm{H}]=0.30-0.40, \tau \sim 7 \mathrm{Gyr})$ that exhibits two prominent overdensities on the horizontal branch (HB). Approximately 45 stars occupy the red clump (RC) region (Buzzoni et al. 2012), which is reproduced by standard stellar evolution codes modeling metal-rich clusters. However, 12 cluster stars-with membership confirmed only for some of them-are significantly hotter than their RC counterparts, which is in conflict with such classic modeling. Those stars are designated extreme HB (EHB) stars when associated with star clusters, and hot subdwarf $\mathrm{B}$ and potentially $\mathrm{O}$ stars when belonging to the field (sdB/sdO). Their effective temperature $T_{\text {eff }}$ and gravity span $T_{\text {eff }}=25,000-45,000 \mathrm{~K}$ and $\log g=4.5-6.2$ (Liebert et al. 1994), respectively. Presumably, these stars are surrounded by a thin hydrogen envelope $\left(\sim 0.01 M_{\odot}\right)$. EHB stars are present in a number of Galactic globular clusters (Moni Bidin et al. 2008). However, first, NGC $6791 \mathrm{HB}$ is much different from any globular cluster HB, as amply discussed in Liebert et al. (1994); second, the combination of mass, age, and metallicity makes NGC 6791 a unique system, with no overlap with Galactic globular clusters.

The mechanism and stellar evolutionary path that give rise to EHB in NGC 6791 have been an active source of debate, particularly since the discovery of a bimodal HB distribution in NGC 6791 (Kaluzny \& Udalski 1992). One proposal involves invoking extreme mass loss that is tied to the cluster's high metallicity (D'Cruz et al. 1996; Yong et al. 2000; Kalirai et al. 2007), and whereby a Reimers (1975) stellar wind massloss parameter as large as $\eta \sim 1.2$ is adopted. Conversely, RC stars exhibit typical masses of $1.03 \pm 0.03 M_{\odot}$ and lose $0.09 \pm 0.07 M_{\odot}$ while ascending the red giant branch (RGB) phase (Miglio et al. 2012), which implies a mass loss compatible with a significantly smaller Reimers parameter of $0.1 \leqslant \eta \leqslant 0.3$. Direct observations confirming a sizable massloss rate (e.g., $\sim 10^{-9} M_{\odot} \mathrm{yr}^{-1}$; Yong et al. 2000) remain outstanding. Asteroseismological studies support a marginal mass-loss rate (Miglio et al. 2012), while direct Spitzer observations did not reveal circumstellar dust production that would accompany enhanced mass loss during the RGB phase

\footnotetext{
${ }^{3}$ Member of the Carrera del Investigador Científico, Comisión de Investigaciones Científicas de la Provincia de Buenos Aires (CIC-PBA).
}

(van Loon et al. 2008). Furthermore, there is a lack of consensus on the details of (fine-tuned) mass loss required to yield the $T_{\text {eff }}$ and envelope size of EHB stars. Finally, current models reproduce nearly all evolutionary phases present in the CMD of NGC 6791 without anomalous mass loss (Carraro \& Chiosi 1995; Buzzoni et al. 2012).

An alternative mechanism reiterated by Liebert et al. (1994) and Carraro et al. (1996) is that EHB stars could emerge from type-B or type-C binary systems, whereby their envelope would be largely removed during a common-envelope (CE) phase (see also Mengel et al. 1976; Han et al. 2002; Brown 2008). Complex models were not initially readily available to provide a robust evaluation of the hypothesis, and moreover, there was little evidence for binarity among the sample. Subsequent observations and models in concert suggest that NGC 6791 exhibits a high binary percentage of $\sim 50 \%$ (Bedin et al. 2008; Twarog et al. 2011), and among the EHB class, three systems have been confirmed: B4 (Pablo et al. 2011; Mochejska et al. 2013), B7, and B8 (Mochejska et al. 2013; van den Berg et al. 2013). Indeed, it has been noted that a high fraction of $\mathrm{sdB}$ stars might belong to binary systems (Green et al. 2001; Maxted 2004 and references therein).

In this study, it is demonstrated that an updated prescription of the Benvenuto \& De Vito (2003) binary evolutionary code successfully predicts the observed and temporal properties of EHB stars in NGC 6791.

\section{Results}

In the following analysis, EHBs are thought to arise from binary evolution, which provides a natural mechanism of depleting the hydrogen-rich outer layer of the star without an ad hoc or simplified prescription of mass loss. Essentially, the mass transfer to the companion is unstable and thus a $\mathrm{CE}$ encompasses the stars; subsequently, as the two stellar nuclei approach each other, the envelope expands owing to heating and is lost (Paczynski 1976; see also the discussion in Maxted 2004).

The binary evolution is modeled via an updated version of the Benvenuto \& De Vito (2003) code, who developed a Henyey-type algorithm to compute stellar evolution in close binary systems, based on a modification of the scheme presented by Kippenhahn et al. (1967) to solve the set of 
differential equations of stellar evolution together with the mass transfer rate. This approach was subsequently modified to ameliorate transporting extremely steep chemical profiles outward (corresponding to stars just prior to undergoing the helium flash). Convection is treated using the canonical mixing length theory with $\alpha_{\mathrm{mlt}}=2.0$, and semi-convection was introduced following Langer et al. (1985) with $\alpha_{\mathrm{sc}}=0.1$.

It is known that diffusion slightly affects $\mathrm{HB}$ evolution (Reed et al. 2012), and it is certainly necessary to account for surface abundances of EHB stars (Michaud et al. 2007). Here, because of the exploratory nature of this Letter, diffusion processes were ignored and will be addressed elsewhere.

Donor stars that evolve to EHB conditions should have initial masses marginally larger than that of cluster turn-off, as EHB stars are undergoing core helium burning, which is an evolutionary phase appreciably shorter than core hydrogen burning. Binary systems consisting of similar mass stars are considered, whereby one star is $1.3 M_{\odot}$, above the turn-off $\left(M_{\mathrm{to}} \approx 1.15 M_{\odot}\right)$, and the companion is slightly below and features a sufficiently lengthy initial orbital period. The stars are modeled with a metallicity of $Z=0.04$. Moreover, EHBs should stem from stars that reached the RGB in the recent past. Consequently, binaries are considered whereby the primaries fill their Roche lobes as they have extended convective envelopes. Such conditions result in systems that undergo a CE stage in which the primary loses the bulk of its hydrogenrich envelope, while the companion keeps its initial mass and the orbital period falls off appreciably. EHB stars are the objects that evolve after emerging from the $\mathrm{CE}$ phase.

Our main interest is not the $\mathrm{CE}$ phase but the emerging objects. Therefore, the CE stage is mimicked assuming a strong mass-loss rate until detachment (Iben \& Tutukov 1993). This causes the deep chemical composition profile to remain essentially unaltered, which is expected since CE lasts a short time. The binary pair is assumed to undergo the CE phase when the helium core reaches mass values of $0.3480,0.3694$, and $0.4067 M_{\odot}$. All of them ignite helium well after the CE phase. Larger helium core masses ignite helium before reaching EHB conditions and delineate an evolutionary path that is unimportant for the present discussion.

Each model was evolved until reaching a radius of detachment $\left(R_{d}\right)$ of 7.5 and $1 R_{\odot}$. For $R_{d}=7.5 R_{\odot}$, the total masses corresponding to each helium core at the end of the CE phase were $0.35048,0.37367$, and $0.41344 M_{\odot}$, whereas for $R_{d}=1 R_{\odot}$ the results were slightly smaller, namely 0.34863 , 0.37084 , and $0.40973 M_{\odot}$. The differences correspond to the varying thickness of the outermost hydrogen layer. After the CE phase, the stars are evolved at constant mass, and the computations are terminated at an age of $\tau \sim 9 \mathrm{Gyr}$, which is an upper limit for the age of NGC 6791.

The evolutionary tracks of the two most massive models for each $R_{d}$ value are presented in Figure 1. As noted above, two radii were assumed after the emergence from the CE phase. The larger $R_{d}$ value implies a thicker hydrogen-rich layer, and thus a lower $T_{\text {eff }}$ during most of the evolution. At post-CE stages, the star evolves blueward and ignites helium off-center owing to strong neutrino emission. The evolutionary track subsequently bends downward almost at constant radius. Thereafter, the star depletes the helium core and then progressively the bottom of helium-rich layers, following a cyclical-like trend. The stars exhibit EHB conditions during that stage (notice the blue squares in Figure 1 that represent the EHB stars in NGC 6791).

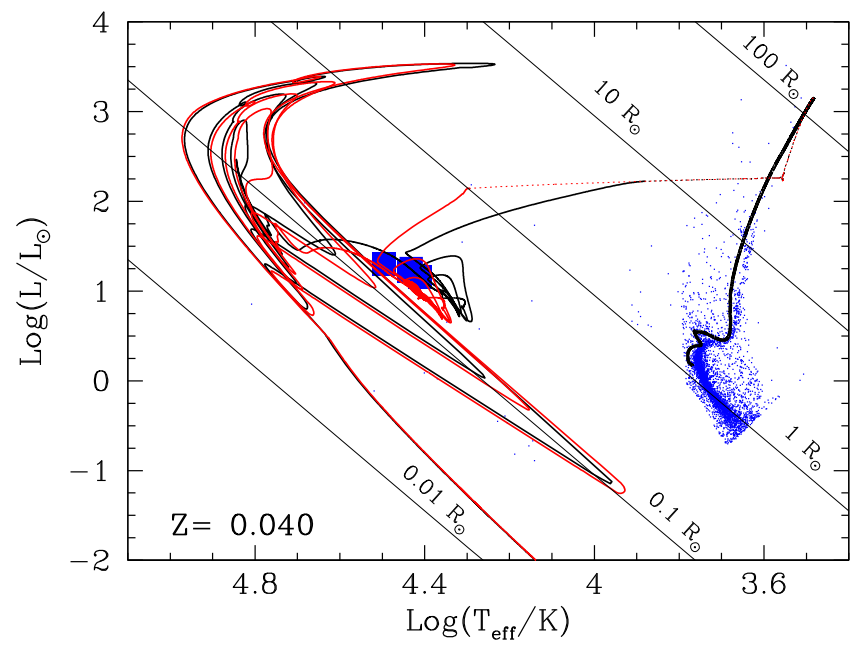

Figure 1. Evolutionary tracks for the initial $1.3 M_{\odot}$ model up to the moment at which it has a helium core with $0.4067 M_{\odot}$. Since then, it is assumed that the object undergoes a $\mathrm{CE}$ episode. If the star emerges from the $\mathrm{CE}$ with a radius of 7.5 (1) $R_{\odot}$, it has a mass of $0.41344(0.40973) M_{\odot}$ and follows the tracks depicted with the black (red) solid line. The blue points are bona fide NGC 6791 members (see Buzzoni et al. 2012), and blue squares represent the EHB stars.

When helium burning becomes weaker, the star contracts, again evolving blueward and igniting the outermost hydrogen layers that gives rise to few thermonuclear flashes. These flashes burn enough hydrogen to cause the star to finally evolve to the white dwarf stage. Lower-mass objects undergo a larger number of cycles during helium burning and hydrogen flashes because nuclear ignition episodes are weaker.

The evolution of stars that emerge from the CE phase with $R_{d}=7.5 R_{\odot}$ is shown in Figure 2, together with data corresponding to the EHB stars B4-B7. Successfully, the model produces $T_{\text {eff }}$ and surface gravities that match the observations. This is largely due to helium ignition that makes the star stop its contraction at the right conditions. As expected, stars that emerge from the CE stage featuring $R_{d}=1 R_{\odot}$ exhibit a larger surface gravity since they are more compact (Figure 3).

It can be noticed from Figure 1 that tracks pass several times across the $T_{\text {eff }}$ interval corresponding to EHBs $\left(\left(\Delta T_{\text {eff }}\right)_{\mathrm{EHB}}\right)$; see Section 1. Most of the time they fall at $\left(\Delta T_{\text {eff }}\right)_{\mathrm{EHB}}$, they undergo helium-burning-dominated cycles. The time they spend at $\left(\Delta T_{\text {eff }}\right)_{\text {EHB }}$ during thermonuclear flashes and the final white dwarf cooling track is much shorter. So, the time the modeled stars spend at $\left(\Delta T_{\text {eff }}\right)_{\mathrm{EHB}}$ is essentially that when they resemble EHBs. This time is crucial since the longer the time the easier to find them as EHBs. Figure 4 conveys the temporal evolution as a function of $T_{\text {eff }}$ for the case of $R_{d}=7.5 R_{\odot}$. Temperature intervals indicated by the observations presented in Liebert et al. (1994) are likewise included. Remarkably, the modeled stars can be detected as EHBs for several hundred million years. The same is true for models featuring $R_{d}=1 R_{\odot}$ (see Figure 5).

\section{Discussion}

The resulting orbital periods of such systems can be estimated via Equation (3) in Ivanova et al. (2013):

$$
\frac{G M_{1} M_{1, \text { env }}}{\lambda R_{1}}=\alpha_{\mathrm{CE}}\left(-\frac{G M_{1} M_{2}}{2 a_{i}}+\frac{G M_{1, c} M_{2}}{2 a_{f}}\right),
$$




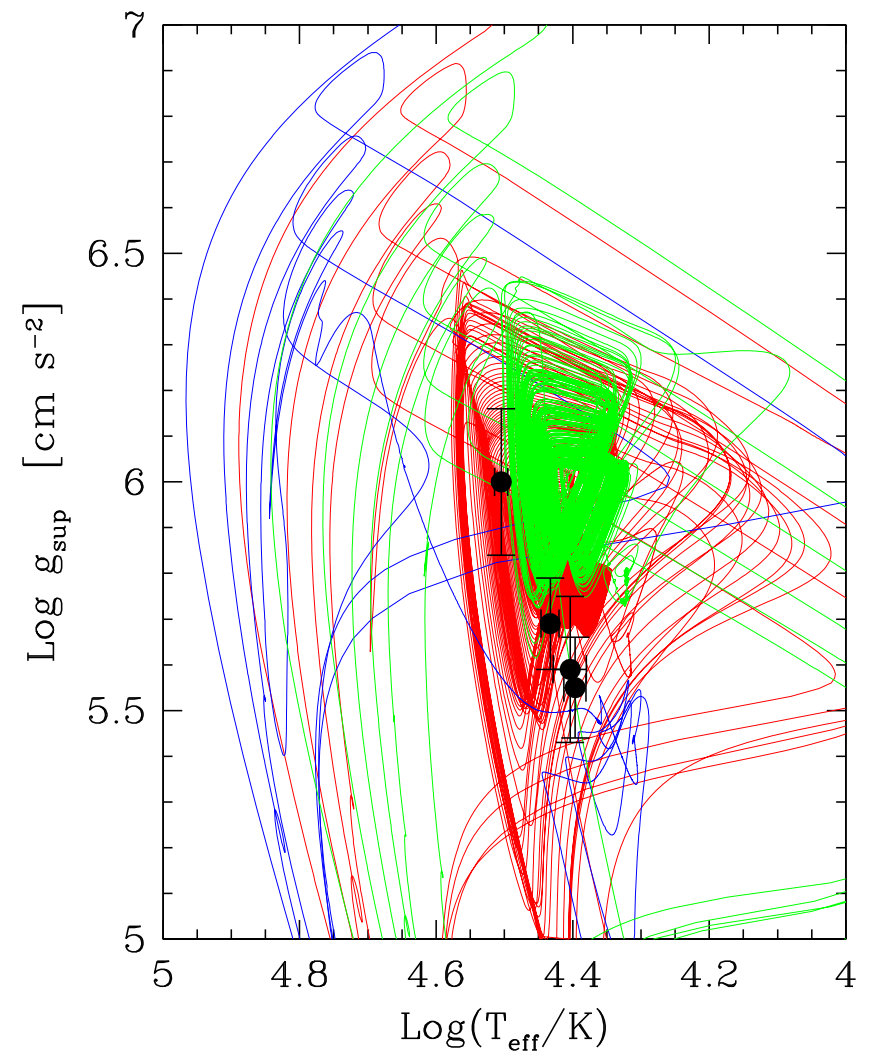

Figure 2. Surface gravity as a function of $T_{\text {eff }}$ for models that detach from CE with $7.5 R_{\odot}$. Green, red, and solid lines correspond to models with masses of $0.35048,0.37367$, and $0.41344 M_{\odot}$, respectively. Data corresponding to stars B3-B6 are shown with their corresponding error bars.

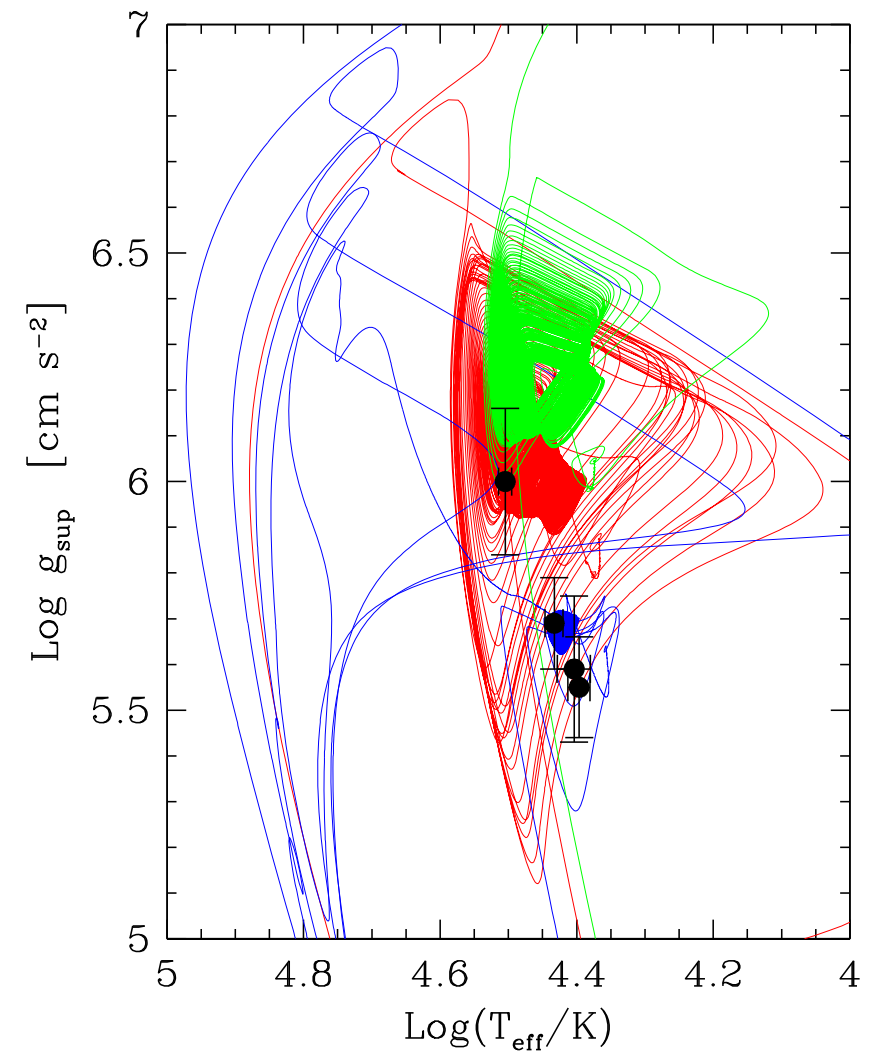

Figure 3. Same as Figure 2, but for the case in which CE resumes at $1 R_{\odot}$. Here, green, red, and blue solid lines correspond to models with masses of $0.34863,0.37084$, and $0.40973 M_{\odot}$, respectively.

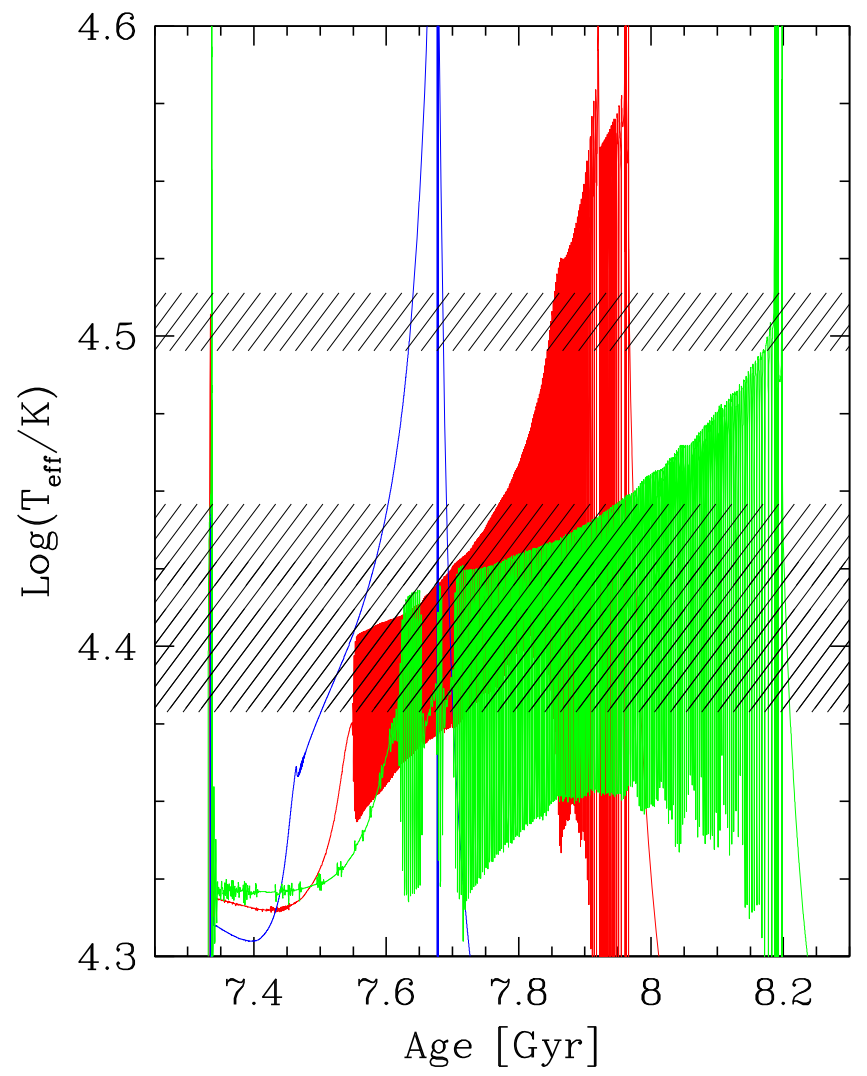

Figure 4. Effective temperature as a function of time for the models included in Figure 2. As in Figure 2, green, red, and blue solid lines correspond to models with masses of $0.35048,0.37367$, and $0.41344 M_{\odot}$, respectively. The hatched areas indicate the $T_{\text {eff }}$ interval (due to error bars) as given in Liebert et al. (1994). B3, B4, and B5 intervals overlap in the lower hatched region, meanwhile the upper one corresponds to star B6. Notice that the $T_{\text {eff }}$ of the models falls inside the observed intervals during a considerable amount of time, making its detection probable.

where $G$ is the gravitational constant and $M_{1}, M_{1, \text { env }}, M_{1, c}$ are the total, envelope, and core masses of the donor star, respectively. $M_{2}$ is the companion mass; $a_{i}, a_{f}$ are the initial and final semi-axes; $\alpha_{\mathrm{CE}}$ is the CE efficiency; and $\lambda$ accounts for the density profile of the donor star. The semi-axis at the onset of mass transfer is $a_{i}$ and is computed via the relation between the orbital semi-axis and the equivalent radius of the Roche lobe (Eggleton 1983). The final orbital period $P_{f}$ follows and is an (increasing) function of the parameter $\xi=\lambda \alpha_{\mathrm{CE}} / 2$. Here, $\xi=0.10$ and $M_{2}=1 M_{\odot}$ are adopted as representative values and the models corresponding to the case of $R_{d}=1 R_{\odot}$.

If Roche-lobe overflow occurs when the donor star develops a helium core of $0.3486 M_{\odot}$ and exhibits a radius of $69 R_{\odot}$, then $a_{i}=172.1 R_{\odot}, a_{f}=1.876 R_{\odot}$, and $P_{f}=0.254$ days. If overflow occurs when the helium core is $0.4067 M_{\odot}$ and features a radius of $137 R_{\odot}$, then $a_{i}=342 R_{\odot}, a_{f}=4.61 R_{\odot}$, and $P_{f}=0.962$ days.

The estimated periods fall in the range of observations (Green et al. 2001; Maxted 2004 and references therein), and indeed, EHB B4 displays an orbital period of $P=0.4$ days (Pablo et al. 2011).

\section{Conclusions}

In this study, it is advocated that binary evolution is the source of the EHB population within NGC 6791, completely 


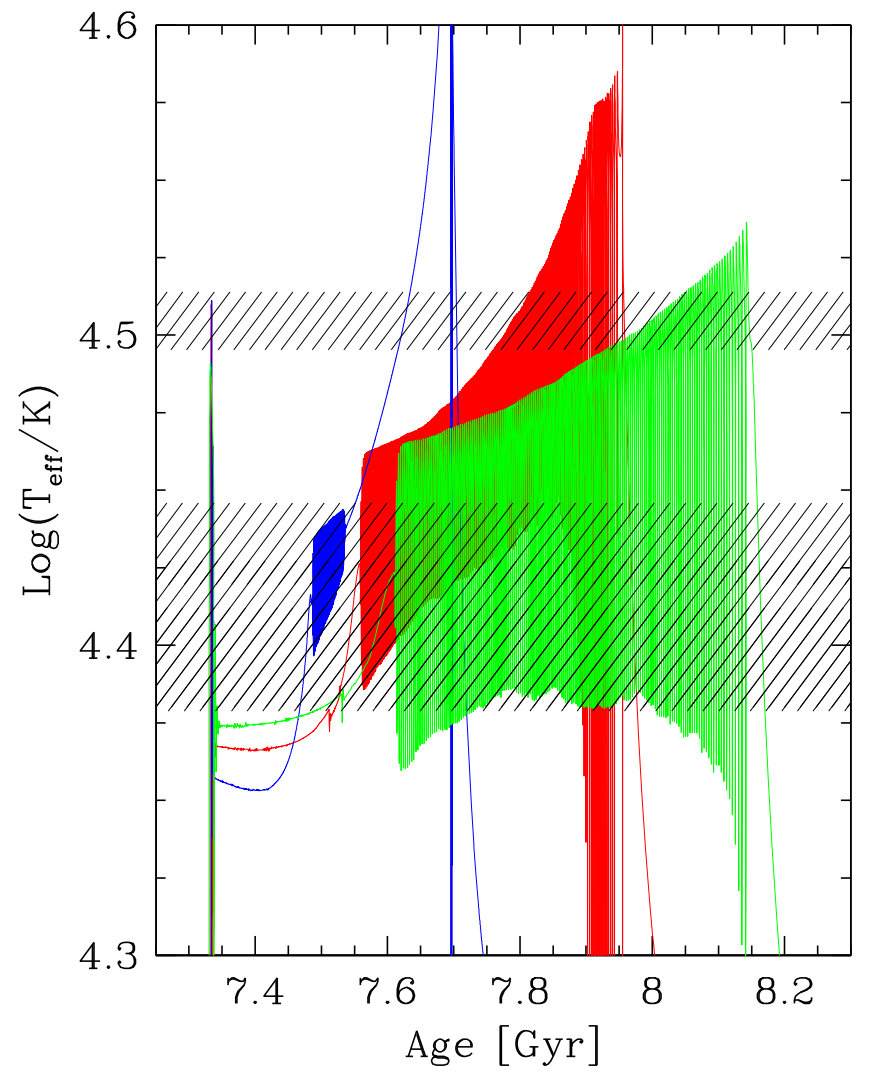

Figure 5. Same as Figure 4, but for the case in which CE resumes at $1 R_{\odot}$.

similar to a field subdwarf (Han et al. 2002). An updated form of the Benvenuto \& De Vito (2003) code is used to demonstrate that EHBs can emerge from the post-CE evolution of binary stars with masses conducive to the cluster's turn-off $\left(M_{\mathrm{to}} \approx 1.15 M_{\odot}\right)$. The numerical model employed yields synthetic stars that match the observational and temporal properties of NGC 6791's EHB members. The binary mechanism is not the only means for stars to evolve to EHB conditions since isolated stars with heavy mass loss might succeed. However, the evolutionary path explored here is preferred since it does not require ad hoc anomalous and observationally unconfirmed mass-loss rates and granted that NGC 6791 and EHB stars exhibit a high rate of binarity.

One may wonder whether our results can be extended to other stellar systems. Unfortunately, no other open cluster is known to harbor EHB stars, which might be interpreted as arguing that they are by far less massive than NGC 6791, even if they host a comparable amount of binary stars. This stresses once again the uniqueness of NGC 6791 among open clusters in the Milky Way. On the other hand, EHBs stars are more common in globular clusters, but they do no share the same properties of the NGC 6791 EHB population (Liebert et al. 1994). First, in NGC 6791, EHBs are not centrally concentrated (Buzzoni et al. 2012), while in globular they are (Liebert et al. 1994). Second, in globulars, they span a much wider range of colors (hence temperature). This was historically interpreted with the existence of a wide range of envelope sizes, hence with differential mass loss during the RGB ascent. Nowadays, the segmented EHB in globulars is mostly interpreted as evidence of multiple stellar generations, each segment with a different degree of $\mathrm{He}$ enhancement (Marino et al. 2014). Other authors consider rapid rotation (Tailo et al. 2015) as well. These scenarios are difficult to invoke for NGC 6791 since we lack any accepted evidence of multiple stellar populations in NGC 6791 (see Geisler et al. 2012; Bragaglia et al. 2014).

G.C. deeply thanks La Plata Observatory for financial support during a visit where this project was started. The authors deeply thank Daniel Majaess for reading and commenting on the manuscript.

\section{References}

Bedin, L. R., Salaris, M., Piotto, G., et al. 2008, ApJL, 679, L29 Benvenuto, O. G., \& De Vito, M. A. 2003, MNRAS, 342, 50 Bragaglia, A., Sneden, C., Carretta, E., et al. 2014, ApJ, 796, 68 Brown, D. 2008, in ASP Conf. Ser. 392, Hot Subdwarf Stars and Related Objects, ed. U. Heber, C. S. Jeffrey, \& R. Napiwotzki (San Francisco, CA: ASP), 83

Buzzoni, A., Bertone, E., Carraro, G., \& Buson, L. 2012, ApJ, 749, 35

Carraro, G., \& Chiosi, C. 1995, in IAA-IAC-University of Pisa Workshop: The Formation of the Milky Way (Heidelberg: ARI), 175

Carraro, G., Girardi, L., Bressan, A., \& Chiosi, C. 1996, A\&A, 305, 849

D’Cruz, N. L., Dorman, B., Rood, R. T., \& O'Connell, R. W. 1996, ApJ, 466, 359

Eggleton, P. P. 1983, ApJ, 268, 368

Geisler, D., Villanova, S., Carraro, G., et al. 2012, ApJL, 756, L40

Green, E. M., Liebert, J., \& Saffer, R. A. 2001, in ASP Conf. Ser. 226, 12th European Workshop on White Dwarfs, ed. J. L. Provencal et al. (San Francisco, CA: ASP), 192

Han, Z., Podsiadlowski, P., Maxted, P. F. L., Marsh, T. R., \& Ivanova, N. 2002, MNRAS, 336, 449

Iben, I., Jr., \& Tutukov, A. V. 1993, ApJ, 418, 343

Ivanova, N., Justham, S., Chen, X., et al. 2013, A\&ARv, 21, 59

Kalirai, J. Zs., Bergeron, P., Hansen, B. M. S., et al. 2007, ApJ, 671, 748

Kaluzny, J., \& Udalski, A. 1992, AcA, 42, 29

Kippenhahn, R., Weigert, A., \& Hofmeister, E. 1967, MComP, 7, 129

Langer, N., El Eid, M. F., \& Fricke, K. J. 1985, A\&A, 145, 179

Liebert, J., Safferm, R. A., \& Green, E. M. 1994, AJ, 107, 1408

Marino, A. F., Milone, A. P., Przybilla, N., et al. 2014, MNRAS, 437, 1609

Maxted, P. F. L. 2004, in ASP Conf. Ser. 318, Spectroscopically and Spatially Resolving the Components of the Close Binary Stars, ed. R. W. Hilditch, H. Hensberge, \& K. Pavlovski (San Francisco, CA: ASP), 387

Mengel, J. G., Norris, J., \& Gross, P. G. 1976, ApJ, 204, 488

Michaud, G., Richer, J., \& Richard, O. 2007, ApJ, 670, 1178

Miglio, A., Brogaard, K., Stello, D., et al. 2012, MNRAS, 419, 2077

Mochejska, B. J., Stanek, K.-Z., \& Kaluzny, J. 2013, AJ, 125, 3175

Moni Bidin, C., Catelan, M., \& Altmann, M. 2008, A\&A, 480, L1

Pablo, H., Kawaler, S. D., \& Green, E. M. 2011, ApJL, 740, L47

Paczynski, B. 1976, in IAU Symp. 73, Structure and Evolution of Close Binary Systems, ed. P. Eggleton, S. Mitton, \& J. Whelan (Dordrecht: D. Reidel), 75

Reed, M. D., Baran, A., Østensen, R. H., Telting, J., \& O’Toole, S. J. 2012, MNRAS, 427, 1245

Reimers, D. 1975, MSRSL, 8, 369

Tailo, M., D'antona, F., Vesperini, E., et al. 2015, Natur, 523, 318

Twarog, B. A., Carraro, G., \& Anthony-Twarog, B. J. 2011, ApJL, 727, L7

van den Berg, M., Verbunt, F., Tagliaferri, G., et al. 2013, ApJ, 770, 98

van Loon, J. Th., Boyer, M. L., \& McDonald, I. 2008, ApJL, 680, L49

Yong, H., Demarque, P., \& Yi, S. 2000, ApJ, 539, 928 